\title{
Existence and stability of Camm type steady states in galactic dynamics
}

\author{
Yan Guo \\ Lefschetz Center for Dynamical Systems \\ Division of Applied Mathematics \\ Brown University, Providence, RI 02912 \\ and \\ Gerhard Rein \\ Mathematisches Institut der Universität München \\ Theresienstr. 39 \\ 80333 München, Germany
}

\begin{abstract}
We prove the existence and nonlinear stability of Camm type steady states of the Vlasov-Poisson system in the gravitational case. The paper demonstrates the effectiveness of an approach to the existence and stability problem for steady states, which was used in previous work by the authors: The steady states are obtained as minimizers of an energy-Casimir functional, and from this fact their dynamical stability is deduced.
\end{abstract}

\section{Introduction}

In astrophysics the Vlasov-Poisson system

$$
\begin{gathered}
\partial_{t} f+v \cdot \nabla_{x} f-\nabla_{x} U \cdot \nabla_{v} f=0, \\
\triangle U=4 \pi \rho,
\end{gathered}
$$




$$
\rho(t, x)=\int f(t, x, v) d v
$$

is used to model the time evolution of a large ensemble of "particles" (stars) which interact only by the gravitational field which they create collectively. Examples of such ensembles are galaxies or globular clusters. Here $f=f(t, x, v) \geq 0$ denotes the density of the particles in phase space, $t \in \mathbb{R}$ denotes time, $x, v \in \mathbb{R}^{3}$ denote position and velocity respectively, $\rho$ is the spatial mass density, and $U$ the gravitational potential. The model does not include relativistic effects - including these would lead to the VlasovEinstein system - or collisions among the particles - these are assumed to be sufficiently rare to be neglected.

In the present paper we are interested in the existence and stability of steady states of this system, and we pursue an approach which has recently been used to construct stable steady states of polytropic type and generalizations of these, cf. [9, 10]. For polytropic steady states the phase space density is of the form

$$
f(x, v)=\left(E_{0}-E\right)_{+}^{k} L^{l} .
$$

Here $(\cdot)_{+}$denotes the positive part, $E_{0} \in \mathbb{R}$ is a constant,

$$
E=\frac{1}{2}|v|^{2}+U(x)
$$

denotes the particle energy which is conserved along characteristics of the Vlasov equation (1.1) if $U$ is time-independent, and

$$
L=|x \times v|^{2}=|v|^{2}|x|^{2}-(x \cdot v)^{2},
$$

denotes the modulus of angular momentum squared which is conserved if $U$ is spherically symmetric. Upon substitution of the ansatz (1.4) into (1.3) the Vlasov-Poisson system is reduced to the - then semilinear-Poisson equation. This approach was followed in [2], where it was shown that solutions of the semilinear Poisson equation exist and lead to steady states with finite mass and compact support, provided $k>-1, l>-1, k+l+3 / 2 \geq 0, k<3 l+7 / 2$. The question whether the resulting steady states are stable is not addressed by this approach. In the present paper we construct steady states as minimizers of an appropriately defined energy-Casimir functional. This has several advantages: The fact that the resulting steady states have finite mass is built into the definition of the set over which one minimizes the energy-Casimir 
functional, and the compact support property is an integral part of the minimization approach as well. Next, the appoach is more flexible in the sense that one does not need an ansatz exactly of the form (1.4), but only certain growth and scaling assumptions. Finally and most importantly, resulting steady states are stable in a well defined sense. In [9] steady states of the form (1.4) with $0<k<l+3 / 2$ were considered, and in 10 this was extended to include steady states of the form $f(x, v)=\phi\left(E_{0}-E, L\right)$ where $\phi$ is characterized by certain growth conditions. An extension of the polytropic ansatz is

$$
f(x, v)=\left(E_{0}-E-\gamma L\right)_{+}^{k} L^{l},
$$

which is due to Camm [5]; here $\gamma \geq 0$ is an additional parameter. We will show that the energy-Casimir technique applies for $k$ and $l$ as above and $\gamma$ small and yields steady states with finite mass and compact support which are nonlinearly stable. At the same time the method will allow a more general dependence on $E_{0}-E-\gamma L$. In the case $\gamma=0$ the present paper includes steady states which were not covered in [9, 10]. As indicated by [2, 5.8], a smallness assumption on $\gamma$ is necessary to obtain steady states with compact support.

The paper proceeds as follows. In the next section we introduce the energy-Casimir functional $\mathcal{D}$ and prove some preliminary results, in particular a lower bound for $\mathcal{D}$ on an appropriate set $\mathcal{F}_{M}$ of test functions with prescribed mass $M$. The crucial part is to show that along a minimizing sequence mass cannot escape to infinity. This is done in Section 3, using the scaling properties of $\mathcal{D}$ and a careful estimate of the contribution of the part of the matter distribution inside and the part outside a given ball in space to the energy-Casimir functional. For this splitting estimate we require spherical symmetry of the functions in $\mathcal{F}_{M}$. In Section 4 we show that a minimizer exists and that any minimizer is a steady state, the latter fact being essentially the Euler-Lagrange identity for our variational problem. In the last section we discuss the stability properties of the resulting steady states.

We conclude this introduction with some further references to the literature. The existence of global classical solutions to the initial value problem for the Vlasov-Poisson system has been shown in 114 as well as in [13, 19. In the monograph [6] one can find many references to discussions of the stability problem in the astrophysics literature. As far as mathematically rigorous results are concerned, we mention [21], where the stability of the polytropes is 
investigated using a variational approach for a reduced energy-Casimir functional defined on the space of mass functions $m(r)=4 \pi \int_{0}^{r} s^{2} \rho(s) d s$, and an investigation of linearized stability in [3]. For the plasma physics case, where the sign in the Poisson equation (1.2) is reversed, the stability problem is much easier and better understood. We refer to [4, 11, 12, 15]. The present approach was also used in [17] to show the existence and stability of extremely flattened steady states which in particular are no longer spherically symmetric. Finally, a very general condition which guarantees finite mass and compact support of steady states, but not their stability, is established in 18 .

\section{Preliminaries; a lower bound for $\mathcal{D}$}

For a measurable, spherically symmetric function $f=f(x, v), x \in \mathbb{R}^{3}$, and $r=|x|$ we define

$$
\rho_{f}(x):=\int f(x, v) d v, m_{f}(x):=\int_{|y| \leq r} \rho_{f}(y) d y,
$$

and

$$
\nabla U_{f}(x):=U_{f}^{\prime}(r) \frac{x}{r}:=\frac{m_{f}(r)}{r^{2}} \frac{x}{r}, U_{f}(r):=-\int_{r}^{\infty} U_{f}^{\prime}(s) d s .
$$

Here spherical symmetry means that

$$
f(A x, A v)=f(x, v), x, v \in \mathbb{R}^{3}, A \in \mathrm{SO}(3) ;
$$

the symmetry is of course only relevant for the definition of the potential. We shall also use the notation $m_{\rho}$ and $U_{\rho}$ if $\rho$ is not necessarily induced by some function $f(x, v)$. Spherically symmetric functions of $x$ will be identified with the corresponding functions of $r=|x|$. Next we define

$$
\begin{aligned}
E_{\mathrm{kin}}(f) & :=\frac{1}{2} \iint|v|^{2} f(x, v) d v d x, \\
E_{\mathrm{pot}}(f) & :=-\frac{1}{8 \pi} \int\left|\nabla U_{f}(x)\right|^{2} d x, \\
\mathcal{C}(f) & :=\iint Q\left(L^{-l} f(x, v)\right) L^{l} d v d x, \\
\mathcal{P}(f) & :=\gamma \int L f(x, v) d v d x+\mathcal{C}(f)+E_{\mathrm{kin}}(f), \\
\mathcal{D}(f) & :=\mathcal{P}(f)+E_{\mathrm{pot}}(f),
\end{aligned}
$$


where $l>-1, Q$ is a given function satisfying certain assumptions specified below, and $\gamma \geq 0$. Note that $\mathcal{P}$ is the positive part of the energy-Casimir functional $\mathcal{D}$. As to the existence of the potential energy part we refer to Lemma 2 below. We will minimize $\mathcal{D}$ over the set

$$
\begin{aligned}
\mathcal{F}_{M}:=\left\{f \in L^{1}\left(\mathbb{R}^{6}\right) \mid\right. & f \geq 0, \iint f d v d x=M, \\
& \mathcal{P}(f)<\infty, \text { and } f \text { is spherically symmetric }\},
\end{aligned}
$$

where $M>0$ is prescribed. The function $Q$ which determines the Casimir functional has to satisfy the following

Assumptions on $Q: Q \in C^{1}\left(\left[0, \infty[) \cap C^{2}(] 0, \infty[), Q \geq 0\right.\right.$, and there exist constants $C_{1}, C_{2}>0, F_{0}>0$, and $0<k_{1}, k_{2}, k_{3}<l+3 / 2$ such that:

(Q1) $Q(f) \geq C_{1} f^{1+1 / k_{1}}, f \geq 0$; if $l=0$ this is required for $f \geq F_{0}$ only.

(Q2) $Q(f) \leq C_{2} f^{1+1 / k_{2}}, 0 \leq f \leq F_{0}$.

(Q3) $Q(\lambda f) \geq \lambda^{1+1 / k_{3}} Q(f), f \geq 0,0 \leq \lambda \leq 1$.

(Q4) $Q^{\prime \prime}(f)>0, f>0$, and $Q^{\prime}(0)=0$.

Remark: The above assumptions imply that $Q^{\prime}$ is strictly increasing with range $[0, \infty[$. On their support the steady states obtained later will be of the form

$$
f_{0}(x, v)=\left(Q^{\prime}\right)^{-1}\left(E_{0}-E-\gamma L\right) L^{l}
$$

with some $E_{0}<0$ and $E$ and $L$ as defined in (1.5) and (1.6) respectively. A typical example of a function $Q$ satisfying the assumptions would be

$$
Q(f)=c_{1} f^{1+1 / k_{1}}+c_{2} f^{1+1 / k_{2}}
$$

with $0<k_{1}, k_{2}<l+3 / 2$ and $c_{1}>0, c_{2} \geq 0$. For $c_{2}=0$ this leads to a steady state of the form (1.7), but this is not so if $c_{2}>0$.

The aim of the present section is to establish a lower bound for $\mathcal{D}$ of a form that will imply the boundedness of $\mathcal{P}$ along any minimizing sequence. On the way we will establish several estimates for $\rho_{f}$ and $U_{f}$ induced by an element $f \in \mathcal{F}_{M}$. 
Lemma 1 (a) There exists a constant $C>0$ such that

$$
\iint f^{1+1 / k_{1}} L^{-l / k_{1}} d v d x \leq C(1+\mathcal{P}(f)), f \in \mathcal{F}_{M}
$$

(b) Let $n_{1}:=k_{1}+l+3 / 2$. Then there exists a constant $C>0$ such that

$$
\int \rho_{f}^{1+1 / n_{1}}|x|^{-2 l / n_{1}} d x \leq C(1+\mathcal{P}(f)), f \in \mathcal{F}_{M} .
$$

Proof. If (Q1) holds with $F_{0}=0$ the estimate in (a) is obvious. If $l=0$ and $F_{0}>0$ one can split the integral according to $f \leq F_{0}$ and $f \geq F_{0}$ and use $M$ to bound the first part and (Q1) to bound the second part. As to (b), we have for any $R>0$ and $x \in \mathbb{R}^{3}$,

$$
\begin{aligned}
\rho_{f}(x) & =\int_{|v| \leq R} f(x, v) d v+\int_{|v| \geq R} f(x, v) d v \\
& \leq\left(\int_{|v| \leq R} L^{l} d v\right)^{1 /\left(1+k_{1}\right)}\left(\int f^{1+1 / k_{1}} L^{-l / k_{1}} d v\right)^{k_{1} /\left(k_{1}+1\right)}+\frac{1}{R^{2}} \int v^{2} f d v \\
& =C|x|^{2 l /\left(k_{1}+1\right)} R^{(2 l+3) /\left(k_{1}+1\right)}\left(\int f^{1+1 / k_{1}} L^{-l / k_{1}} d v\right)^{k_{1} /\left(k_{1}+1\right)}+\frac{1}{R^{2}} \int v^{2} f d v \\
& \leq C|x|^{2 l /\left(k_{1}+l+5 / 2\right)}\left(\int f^{1+1 / k_{1}} L^{-l / k_{1}} d v+\int v^{2} f d v\right)^{n_{1} /\left(n_{1}+1\right)}
\end{aligned}
$$

by Hölder's inequality and optimization in $R$. Taking both sides of the inequality to the power $1+1 / n_{1}$, dividing by $|x|^{2 l / n_{1}}$, and integrating with respect to $x$ yields the assertion.

Motivated by Lemma 11 we define

$$
\begin{gathered}
L^{k_{1}, l}\left(\mathbb{R}^{6}\right):=\left\{f: \mathbb{R}^{6} \rightarrow \mathbb{R} \mid f\right. \text { measurable, spherically symmetric, and } \\
\left.\iint f^{1+1 / k_{1}} L^{-l / k_{1}} d v d x<\infty\right\}
\end{gathered}
$$

equipped with the norm

$$
\|f\|_{k_{1}, l}:=\left(\iint f^{1+1 / k_{1}} L^{-l / k_{1}} d v d x\right)^{k_{1} /\left(k_{1}+1\right)}
$$

and

$$
\begin{gathered}
L^{n_{1}, l}\left(\mathbb{R}^{3}\right):=\left\{\rho: \mathbb{R}^{3} \rightarrow \mathbb{R} \mid \rho\right. \text { measurable, spherically symmetric, and } \\
\left.\qquad \rho^{1+1 / n_{1}}|x|^{-2 l / n_{1}} d x<\infty\right\}
\end{gathered}
$$


equipped with the norm

$$
\|\rho\|_{n_{1}, l}:=\left(\int \rho^{1+1 / n_{1}}|x|^{-2 l / n_{1}} d x\right)^{n_{1} /\left(n_{1}+1\right)} .
$$

Both spaces are reflexive Banach spaces.

Lemma 2 (a) There exist constants $C>0$ and $q>0$ such that for $\rho \in$ $L^{n_{1}, l}\left(\mathbb{R}^{3}\right)$ with $\int|\rho|=M$ we have

$$
\begin{aligned}
\int\left|\nabla U_{\rho}\right|^{2} d x & \leq 4 \pi \int_{0}^{R} \frac{m_{\rho}^{2}(r)}{r^{2}} d r+\frac{4 \pi M^{2}}{R} \\
& \leq C R^{q}\left(1+\|\rho\|_{n_{1}, l}^{1+1 / n_{1}}\right)+\frac{4 \pi M^{2}}{R}, R>0
\end{aligned}
$$

(b) For every $R>0$ the mapping

$$
T:\left.L^{n_{1}, l}\left(\mathbb{R}^{3}\right) \ni \rho \mapsto \frac{m_{\rho}}{r}\right|_{[0, R]} \in L^{2}([0, R])
$$

is compact.

(c) For $\rho_{1}, \rho_{2} \in L^{n_{1}, l}\left(\mathbb{R}^{3}\right) \cap L^{1}\left(\mathbb{R}^{3}\right)$ the following identity holds:

$$
\int \nabla U_{\rho_{1}} \cdot \nabla U_{\rho_{2}} d x=-4 \pi \int U_{\rho_{1}} \rho_{2} d x
$$

Proof. Hölder's inequality implies that for any $\rho \in L^{n_{1}, l}\left(\mathbb{R}^{3}\right)$,

$$
\left|m_{\rho}(r)\right| \leq(4 \pi)^{1 /\left(1+n_{1}\right)}\|\rho\|_{n_{1}, l} r^{(2 l+3) /\left(n_{1}+1\right)}, r \geq 0 \text {, }
$$

and thus

$$
\int_{0}^{R} \frac{m_{\rho}^{2}(r)}{r^{2}} d r \leq C\|\rho\|_{n_{1}, l}^{2} R^{\left(4 l+5-n_{1}\right) /\left(n_{1}+1\right)} .
$$

The first estimate for $\nabla U_{\rho}$ in (a) follows from spherical symmetry and the fact that $\left|m_{\rho}\right| \leq M$. For $n_{1} \leq 1$ the second estimate immediately follows from (2.4). For $n_{1}>1$ we use $\left|m_{\rho}\right| \leq M$ and (2.3) to obtain

$$
\int_{0}^{R} \frac{m_{\rho}^{2}(r)}{r^{2}} d r \leq M^{1-1 / n_{1}} \int_{0}^{R} \frac{\left|m_{\rho}\right|^{1+1 / n_{1}}(r)}{r^{2}} d r \leq C\|\rho\|_{n_{1}, l}^{1+1 / n_{1}} R^{\left(2 l+3-n_{1}\right) / n_{1}} .
$$


Since in both cases the power of $R$ is positive, this proves (a). As to (b), we first observe that the operator $T$ is bounded by (2.4). To show its compactness we take a bounded set $S \subset L^{n_{1}, l}\left(\mathbb{R}^{3}\right)$ and apply the Frechét-Kolmogorov criterion to the set $T S$. We redefine $T \rho:=\frac{m_{\rho}}{r} 1_{[0, R]} \in L^{2}(\mathbb{R})$ where $1_{[0, R]}$ is the characteristic function of the interval $[0, R]$. The crucial part is so show that

$$
\left\|(T \rho)_{h}-T \rho\right\|_{2} \rightarrow 0, h \rightarrow 0
$$

uniformly in $\rho \in S$, where $(T \rho)_{h}=(T \rho)(\cdot+h)$. For $h>0$ this follows from the estimate

$$
\begin{aligned}
\left\|\left(\frac{m_{\rho}}{r} 1_{[0, R]}\right)_{h}-\frac{m_{\rho}}{r} 1_{[0, R]}\right\|_{2}^{2} \leq & 2 \int_{0}^{h} \frac{m_{\rho}^{2}}{r^{2}} d r+\int_{R-h}^{R} \frac{m_{\rho}^{2}}{r^{2}} d r \\
& +\int_{h}^{R-h} m_{\rho}^{2}(r)\left|\frac{1}{r+h}-\frac{1}{r}\right|^{2} d r \\
& +\int_{h}^{R-h} \frac{1}{(r+h)^{2}}\left|m_{\rho}(r+h)-m_{\rho}(r)\right|^{2} d r .
\end{aligned}
$$

For the first three terms one uses the estimate (2.3). By Hölder's inequality

$$
\left|m_{\rho}(r+h)-m_{\rho}(r)\right| \leq C\|\rho\|_{n_{1}, l}\left((r+h)^{2 l+3}-r^{2 l+3}\right)^{1 /\left(n_{1}+1\right)},
$$

and together with Lebesgue's dominated convergence theorem this yields the convergence of the last term. Obviously, each term converges uniformly in $\rho \in S$, and the case $h<0$ is completely analogous. As to part (c), we have

$$
\begin{aligned}
\int \nabla U_{\rho_{1}} \cdot \nabla U_{\rho_{2}} d x & =4 \pi \int_{0}^{\infty} U_{\rho_{1}}^{\prime}(r) m_{\rho_{2}}(r) d r \\
& =\left.4 \pi U_{\rho_{1}}(r) m_{\rho_{2}}(r)\right|_{r=0} ^{r=\infty}-(4 \pi)^{2} \int_{0}^{\infty} U_{\rho_{1}}(r) r^{2} \rho_{2}(r) d r \\
& =-4 \pi \int U_{\rho_{1}} \rho_{2} d x
\end{aligned}
$$

Here the boundary term at infinity vanishes since $\left|U_{\rho_{1}}(r)\right| \leq\left\|\rho_{1}\right\|_{1} / r$ and $\left|m_{\rho_{2}}(r)\right| \leq\left\|\rho_{2}\right\|_{1}$, and the boundary term at zero vanishes by (2.3).

Lemma 3 There exists a constant $C>0$ such that

$$
\mathcal{D}(f) \geq \frac{1}{2} \mathcal{P}(f)-C, f \in \mathcal{F}_{M},
$$

in particular,

$$
\mathcal{D}_{M}:=\inf \left\{\mathcal{D}(f) \mid f \in \mathcal{F}_{M}\right\}>-\infty
$$


Proof. Using the previous two lemmas we have

$$
\begin{aligned}
\mathcal{D}(f) & \geq \mathcal{P}(f)-C R^{q}\left(1+\left\|\rho_{f}\right\|_{n_{1}, l}^{1+1 / n_{1}}\right)-\frac{4 \pi M^{2}}{R} \\
& \geq \mathcal{P}(f)\left(1-C R^{q}\right)-C R^{q}-\frac{4 \pi M^{2}}{R},
\end{aligned}
$$

where $C>0$ is some constant which does not depend on $R>0$. The assertion follows by a suitable choice of $R$.

\section{$3 \quad$ Scaling and splitting}

The behaviour of $\mathcal{D}$ and $M$ under scaling transformations can be used to show that $\mathcal{D}_{M}$ is negative for $\gamma$ small and to relate the $\mathcal{D}_{M}$ 's for different values of $M$ :

Lemma 4 (a) Let $M>0$. Then $-\infty<\mathcal{D}_{M}<0$ for $\gamma \geq 0$ sufficiently small.

(b) There exists $\alpha>0$ such that for all $\gamma \geq 0$ and $0<M_{1} \leq M_{2}$,

$$
\mathcal{D}_{M_{1}} \geq\left(\frac{M_{1}}{M_{2}}\right)^{1+\alpha} \mathcal{D}_{M_{2}} .
$$

Proof. Given any function $f$, we define a rescaled function $\bar{f}(x, v)=$ $a f(b x, c v)$, where $a, b, c>0$. Then

$$
\iint \bar{f} d v d x=a b^{-3} c^{-3} \iint f d v d x
$$

and

$$
\begin{aligned}
\mathcal{D}(\bar{f})= & \gamma a b^{-5} c^{-5} \iint L f d v d x+b^{-3-2 l} c^{-3-2 l} \mathcal{C}\left(a b^{2 l} c^{2 l} f\right) \\
& +a b^{-3} c^{-5} E_{\text {kin }}(f)+a^{2} b^{-5} c^{-6} E_{\mathrm{pot}}(f) .
\end{aligned}
$$

To prove (a) we fix some $f \in \mathcal{F}_{1}$ with compact support and $L^{-l} f \leq F_{0}$. Let

$$
a=M b^{3} c^{3}
$$

so that

$$
\iint \bar{f} d v d x=M
$$


Using (Q2),

$$
\mathcal{D}(\bar{f}) \leq \bar{C}_{1} \gamma(b c)^{-2}+\bar{C}_{2} a^{1 / k_{2}}(b c)^{2 l / k_{2}}+\bar{C}_{3} c^{-2}-\bar{C}_{4} b
$$

where $\bar{C}_{1}, \ldots, \bar{C}_{4}>0$ depend on $f$, and we need to make sure that $a(b c)^{2 l} \leq 1$ so that (Q2) applies. Since we want the last term to dominate as $b \rightarrow 0$, we let $c=b^{-\eta / 2}$ so that $b c=b^{1-\eta / 2}$ for some $\eta>0$. Then

$$
\mathcal{D}(\bar{f}) \leq \bar{C}_{1} \gamma b^{\eta-2}+\bar{C}_{2} b^{(1-\eta / 2)(2 l+3) / k_{2}}+\bar{C}_{3} b^{\eta}-\bar{C}_{4} b .
$$

Now fix $\eta \in] 1,2\left[\right.$ such that $(1-\eta / 2)(2 l+3) / k_{2}>1$; such an $\eta$ exists by the assumptions on $k_{2}$ and $l$. For $b>0$ sufficiently small the sum of the last three terms will be negative and $a(b c)^{2 l}=M b^{(3+2 l)(1-\eta / 2)}<1$. If we fix such a $b$ then all the parameters in the above estimate are determined in terms of $M$, except for $\gamma$ which now can be chosen sufficiently small to guarantee that the right hand side of the estimate above is negative.

To show part (b) we assume that $f \in \mathcal{F}_{M_{2}}$ and $\bar{f} \in \mathcal{F}_{M_{1}}$ so that by (3.1),

$$
a b^{-3} c^{-3}=\frac{M_{1}}{M_{2}}=: m \leq 1 .
$$

By (3.2) and (Q3),

$$
\begin{aligned}
\mathcal{D}(\bar{f})= & \gamma m(b c)^{-2} \iint L f \\
& +m a^{-1}(b c)^{-2 l} \mathcal{C}\left(a(b c)^{2 l} f\right)+m c^{-2} E_{\mathrm{kin}}(f)+m^{2} b E_{\mathrm{pot}}(f) \\
\geq & \gamma m(b c)^{-2} \iint L f+m a^{1 / k_{3}}(b c)^{2 l / k_{3}} \mathcal{C}(f)+m c^{-2} E_{\mathrm{kin}}(f)+m^{2} b E_{\mathrm{pot}}(f),
\end{aligned}
$$

provided $a(b c)^{2 l} \leq 1$. Now we require that

$$
m a^{1 / k_{3}}(b c)^{2 l / k_{3}}=m c^{-2}=m^{2} b .
$$

Together with (3.3) this determines $a, b, c$ in terms of $m$. In particular $a(b c)^{2 l}=m^{2 k_{3}(1+l) /\left(3 / 2+l-k_{3}\right)} \leq 1$. We have

$$
\begin{aligned}
\mathcal{D}(\bar{f}) & \geq \gamma m(b c)^{-2} \int L f+m^{1+\alpha}\left(\mathcal{C}(f)+E_{\mathrm{kin}}(f)+E_{\mathrm{pot}}(f)\right) \\
& \geq m^{1+\alpha} \mathcal{D}(f)
\end{aligned}
$$


where $\alpha=(2 l+2) /\left(l+3 / 2-k_{3}\right)>0$; observe that $m(b c)^{-2} \geq m^{1+\alpha}$ since $b c=$ $m^{(l+1) /\left(l+3 / 2-k_{3}\right)-1}$. Since for any given choice of $a, b, c$ the mapping $f \mapsto \bar{f}$ is one-to-one and onto between $\mathcal{F}_{M_{2}}$ and $\mathcal{F}_{M_{1}}$ the scaling inequality follows.

The scaling estimate above can be used to show that along a minimizing sequence the mass has to concentrate in a certain ball:

Lemma 5 Let $M>0$, and let $\gamma \geq 0$ be sufficiently small so that Lemma 4 (a) applies. Then there exists a radius $R_{M}>0$ such that if $\left(f_{n}\right) \subset \mathcal{F}_{M}$ is a minimizing sequence of $\mathcal{D}$,

$$
\lim _{n \rightarrow \infty} \int_{|x| \geq R} \int f_{n} d v d x=0, R>R_{M}
$$

Proof. We define the ball $B_{R}:=\left\{x \in \mathbb{R}^{3}|| x \mid \leq R\right\}$. Let $1_{B_{R} \times \mathbb{R}^{3}}$ be the characteristic function of $B_{R} \times \mathbb{R}^{3}$. For $f \in \mathcal{F}_{M}$ we split

$$
f_{1}=1_{B_{R} \times \mathbb{R}^{3}} f, f_{2}=f-f_{1}
$$

and let $\rho_{i}$ and $U_{i}$ denote the induced spatial densities and potentials respectively, $i=1,2$. We abbreviate $\lambda=M-m_{f}(R)$. Then

$$
\begin{aligned}
\mathcal{D}(f) & =\mathcal{D}\left(f_{1}\right)+\mathcal{D}\left(f_{2}\right)-\frac{1}{4 \pi} \int \nabla U_{1} \cdot \nabla U_{2} \\
& \geq \mathcal{D}_{M-\lambda}+\mathcal{D}_{\lambda}-\frac{1}{4 \pi} \int \nabla U_{1} \cdot \nabla U_{2}
\end{aligned}
$$

since $f_{1} \in \mathcal{F}_{M-\lambda}$ and $f_{2} \in \mathcal{F}_{\lambda}$. Since $\nabla U_{2}=0$ on $B_{R}$,

$$
\int \nabla U_{1} \cdot \nabla U_{2} \leq \lambda(M-\lambda) 4 \pi \int_{R}^{\infty} \frac{1}{r^{2}} d r=\frac{4 \pi}{R} \lambda(M-\lambda) .
$$

Using Lemma 4 (b) we find that

$$
\mathcal{D}(f) \geq\left[(1-\lambda / M)^{1+\alpha}+(\lambda / M)^{1+\alpha}\right] \mathcal{D}_{M}-\frac{1}{R} \lambda(M-\lambda) .
$$

Since $\alpha>0$, there is $C_{\alpha}>0$, such that

$$
(1-x)^{1+\alpha}+x^{1+\alpha}-1 \leq-C_{\alpha}(1-x) x, 0 \leq x \leq 1 .
$$




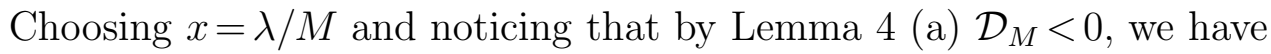

$$
\begin{aligned}
\mathcal{D}(f)-\mathcal{D}_{M} & \geq\left[(1-\lambda / M)^{1+\alpha}+(\lambda / M)^{1+\alpha}-1\right] \mathcal{D}_{M}-\frac{1}{R} \lambda(M-\lambda) \\
& \geq-C_{\alpha} \mathcal{D}_{M}\left(1-\frac{\lambda}{M}\right) \frac{\lambda}{M}-\frac{1}{R} \lambda(M-\lambda) \\
& =\left(-\frac{C_{\alpha} \mathcal{D}_{M}}{M^{2}}-\frac{1}{R}\right)(M-\lambda) \lambda \\
& =\left(\frac{1}{R_{M}}-\frac{1}{R}\right) m_{f}(R)\left(M-m_{f}(R)\right)
\end{aligned}
$$

where

$$
R_{M}:=-\frac{M^{2}}{C_{\alpha} \mathcal{D}_{M}}>0
$$

Now let $\left(f_{n}\right) \subset \mathcal{F}_{M}$ be a minimizing sequence of $\mathcal{D}$, and assume the assertion of the lemma is wrong. Then there exist some $R>R_{M}, \lambda>0$, and a subsequence, called $\left(f_{n}\right)$ again, such that

$$
\lim _{n \rightarrow \infty} \int_{|x| \geq R} \int f_{n} d v d x=\lambda
$$

For every $n \in \mathbb{N}$ we can choose $R_{n}>R$ such that

$$
\lambda_{n}:=\int_{|x| \geq R_{n}} \int f_{n} d v d x=\frac{1}{2} \int_{|x| \geq R} \int f_{n} d v d x .
$$

Then

$$
\lim _{n \rightarrow \infty} \int_{|x| \geq R_{n}} \int f_{n} d v d x=\lim _{n \rightarrow \infty} \lambda_{n}=\lambda / 2>0 .
$$

Applying the estimate (3.4) to $B_{R_{n}}$ we get

$$
\begin{aligned}
\mathcal{D}\left(f_{n}\right)-\mathcal{D}_{M} & \geq\left(\frac{1}{R_{M}}-\frac{1}{R_{n}}\right)\left(M-\lambda_{n}\right) \lambda_{n}>\left(\frac{1}{R_{M}}-\frac{1}{R}\right)\left(M-\lambda_{n}\right) \lambda_{n} \\
& \rightarrow\left(\frac{1}{R_{M}}-\frac{1}{R}\right)(M-\lambda / 2) \lambda / 2>0, n \rightarrow \infty
\end{aligned}
$$

since $0<\lambda / 2<M$. This contradicts the fact that $\left(f_{n}\right)$ is a minimizing sequence. 


\section{Minimizers of $\mathcal{D}$}

Theorem 1 Let $M>0$, and let $\gamma \geq 0$ be sufficiently small so that Lemma 4 (a) applies. Let $\left(f_{n}\right) \subset \mathcal{F}_{M}$ be a minimizing sequence of $\mathcal{D}$. Then there is a minimizer $f_{0}$ and a subsequence $\left(f_{n_{k}}\right)$ such that $\mathcal{D}\left(f_{0}\right)=\mathcal{D}_{M}$, supp $f_{0} \subset$ $B_{R_{M}} \times \mathbb{R}^{3}$ with $R_{M}$ as in Lemma 5, and $f_{n_{k}} \rightarrow f_{0}$ weakly in $L^{k_{1}, l}\left(\mathbb{R}^{6}\right)$. For the induced potentials we have $\nabla U_{n_{k}} \rightarrow \nabla U_{0}$ strongly in $L^{2}\left(\mathbb{R}^{3}\right)$.

Proof. By Lemma 3, $\left(\mathcal{P}\left(f_{n}\right)\right)$ and thus $\left(f_{n}\right)$ is bounded in $L^{k_{1}, l}\left(\mathbb{R}^{6}\right)$, cf. Lemma 1. Thus there exists a weakly convergent subsequence, denoted by $\left(f_{n}\right)$ again:

$$
f_{n} \rightarrow f_{0} \text { weakly in } L^{k_{1}, l}\left(\mathbb{R}^{6}\right) .
$$

Clearly, $f_{0} \geq 0$ a. e., and $f_{0}$ is spherically symmetric. Since by Lemma 5

$$
\begin{aligned}
M & =\lim _{n \rightarrow \infty} \int_{|x| \leq R_{1}} \int_{|v| \leq R_{2}} f_{n} d v d x+\lim _{n \rightarrow \infty} \int_{|x| \leq R_{1}} \int_{|v| \geq R_{2}} f_{n} d v d x \\
& \leq \lim _{n \rightarrow \infty} \int_{|x| \leq R_{1}} \int_{|v| \leq R_{2}} f_{n} d v d x+\frac{C}{R_{2}^{2}}
\end{aligned}
$$

where $R_{1}>R_{M}$ and $R_{2}>0$ are arbitrary, it follows that

$$
\int_{|x| \leq R_{1}} \int f_{0} d v d x=M
$$

for every $R_{1}>R_{M}$. This proves the assertion on $\operatorname{supp} f_{0}$ and $\iint f_{0}=M$. Also by weak convergence

$$
\iint|v|^{2} f_{0} d v d x \leq \liminf _{n \rightarrow \infty} \iint|v|^{2} f_{n} d v d x<\infty .
$$

By Lemma $1\left(\rho_{n}\right)=\left(\rho_{f_{n}}\right)$ is bounded in $L^{n_{1}, l}\left(\mathbb{R}^{3}\right)$. After extracting a further subsequence, we thus have that

$$
\rho_{n} \rightarrow \rho_{0}:=\rho_{f_{0}} \text { weakly in } L^{n_{1}, l}\left(\mathbb{R}^{3}\right) .
$$

Thus by Lemma 2 the convergence of the fields in $L^{2}\left(\mathbb{R}^{3}\right)$ follows.

It remains to show that $f_{0}$ is actually a minimizer, in particular, $\mathcal{P}\left(f_{0}\right)<\infty$ so that $f_{0} \in \mathcal{F}_{M}$. By Mazur's Lemma there exists a sequence $\left(g_{n}\right) \subset L^{k_{1}, l}\left(\mathbb{R}^{6}\right)$ such that $g_{n} \rightarrow f_{0}$ strongly in $L^{k_{1}, l}\left(\mathbb{R}^{6}\right)$ and $g_{n}$ is a convex 
combination of $\left\{f_{k} \mid k \geq n\right\}$. In particular, $g_{n} \rightarrow f_{0}$ a. e. on $\mathbb{R}^{6}$. By (Q4) the functional

$$
f \mapsto \iint\left(\gamma L f+Q\left(L^{-l} f\right) L^{l}\right) d v d x
$$

is convex. Combining this with Fatou's Lemma implies that

$$
\begin{aligned}
\iint\left(\gamma L f_{0}+Q\left(L^{-l} f_{0}\right) L^{l}\right) d v d x & \leq \liminf _{n \rightarrow \infty} \iint\left(\gamma L g_{n}+Q\left(L^{-l} g_{n}\right) L^{l}\right) d v d x \\
& \leq \limsup _{n \rightarrow \infty} \iint\left(\gamma L f_{n}+Q\left(L^{-l} f_{n}\right) L^{l}\right) d v d x .
\end{aligned}
$$

Together with (4.1) this implies that

$$
\mathcal{P}\left(f_{0}\right) \leq \lim _{n \rightarrow \infty} \mathcal{P}\left(f_{n}\right)<\infty
$$

note that $\lim _{n \rightarrow \infty} \mathcal{P}\left(f_{n}\right)$ exists. Therefore,

$$
\mathcal{D}\left(f_{0}\right)=\mathcal{P}\left(f_{0}\right)-\frac{1}{8 \pi} \int\left|\nabla U_{0}\right|^{2} \leq \lim _{n \rightarrow \infty}\left(\mathcal{P}\left(f_{n}\right)-\frac{1}{8 \pi} \int\left|\nabla U_{n}\right|^{2}\right)=\mathcal{D}_{M},
$$

and the proof is complete.

Theorem 2 Let $f_{0} \in \mathcal{F}_{M}$ be a minimizer of $\mathcal{D}$. Then

$$
f_{0}(x, v)=\left\{\begin{array}{cl}
\left(Q^{\prime}\right)^{-1}\left(E_{0}-E-\gamma L\right) L^{l} & , E_{0}-E-\gamma L>0, \\
0 & , E_{0}-E-\gamma L \leq 0
\end{array}\right.
$$

where

$$
\begin{gathered}
E:=\frac{1}{2}|v|^{2}+U_{0}(x), \\
E_{0}:=\frac{1}{M} \iint\left(Q^{\prime}\left(f_{0}\right)+E+\gamma L\right) f_{0} d v d x<0
\end{gathered}
$$

and $U_{0}$ is the potential induced by $f_{0}$. Moreover, $f_{0}$ is a steady state of the Vlasov-Poisson system.

Proof. Let $f_{0}$ be a minimizer. We shall use the standard method of EulerLagrange multipliers to prove the theorem. Let $\epsilon>0$, and $\eta \in L^{\infty}\left(\mathbb{R}^{6}\right)$ be compactly supported and spherically symmetric with

$$
\eta \geq 0 \text { a. e. on } \mathbb{R}^{6} \backslash \operatorname{supp} f_{0}, \iint \eta d v d x=0,
$$




$$
\epsilon \leq f_{0} \leq \frac{1}{\epsilon} \text { a. e. on } \operatorname{supp} f_{0} \cap \operatorname{supp} \eta, \epsilon \leq L \leq \frac{1}{\epsilon} \text { a. e. on } \operatorname{supp} \eta \text {. }
$$

Below we will occasionally argue pointwise on $\mathbb{R}^{6}$ so we choose a representative of $f_{0}$ satisfying the previous estimate pointwise. For

$$
0 \leq h \leq \frac{\epsilon}{2\left(1+\|\eta\|_{\infty}\right)}
$$

we have $f_{0}+h \eta \in \mathcal{F}_{M}$; that $\mathcal{C}\left(f_{0}+h \eta\right)<\infty$ will follow from the estimates below. We expand $\mathcal{D}\left(f_{0}+h \eta\right)-\mathcal{D}\left(f_{0}\right)$ in powers of $h$ :

$$
\begin{aligned}
\mathcal{D}\left(f_{0}+h \eta\right)-\mathcal{D}\left(f_{0}\right)= & \iint\left(Q\left(L^{-l}\left(f_{0}+h \eta\right)\right)-Q\left(L^{-l} f_{0}\right)\right) L^{l} d v d x \\
& +h \iint\left(\gamma L+\frac{1}{2}|v|^{2}+U_{0}\right) \eta d v d x-h^{2} \frac{1}{8 \pi} \int\left|\nabla U_{\eta}\right|^{2} d x ;
\end{aligned}
$$

in expanding the potential energy term we used Lemma 2 (c). To expand the first term we first consider a point $(x, v) \in \operatorname{supp} f_{0} \cap \operatorname{supp} \eta$. Then

$$
\left(Q\left(L^{-l}\left(f_{0}+h \eta\right)\right)-Q\left(L^{-l} f_{0}\right)\right) L^{l}=h Q^{\prime}\left(L^{-l} f_{0}\right) \eta+h^{2} \frac{1}{2} Q^{\prime \prime}(\tau) L^{-l} \eta^{2}
$$

where $\tau$ lies between $L^{-l} f_{0}$ and $L^{-l}\left(f_{0}+h \eta\right)$. Thus

$$
\left|\left(Q\left(L^{-l}\left(f_{0}+h \eta\right)\right)-Q\left(L^{-l} f_{0}\right)\right) L^{l}-h Q^{\prime}\left(L^{-l} f_{0}\right) \eta\right| \leq C h^{2} \eta^{2} ;
$$

note that $0<c_{1} \leq L^{-l} f_{0}, L^{-l}\left(f_{0}+h \eta\right) \leq c_{2}$ on $\operatorname{supp} \eta \cap \operatorname{supp} f_{0}$ for constants $c_{1}, c_{2}>0$, and $Q^{\prime \prime}$ is continuous on the interval $\left[c_{1}, c_{2}\right]$. On $\operatorname{supp} \eta \backslash \operatorname{supp} f_{0}$ the assumption (Q2) implies that for $h$ small

$$
\begin{aligned}
\left|\left(Q\left(L^{-l}\left(f_{0}+h \eta\right)\right)-Q\left(L^{-l} f_{0}\right)\right) L^{l}-h Q^{\prime}\left(L^{-l} f_{0}\right) \eta\right| & =Q\left(L^{-l} h \eta\right) L^{l} \\
& \leq C|\eta|^{1+1 / k_{2}} h^{1+1 / k_{2}} .
\end{aligned}
$$

The fact that $f_{0}$ is a minimizer and the estimates above imply that

$$
\begin{aligned}
0 \leq \mathcal{D}\left(f_{0}+h \eta\right)-\mathcal{D}\left(f_{0}\right)= & h \iint\left(Q^{\prime}\left(L^{-l} f_{0}\right)+\gamma L+\frac{1}{2}|v|^{2}+U_{0}\right) \eta d v d x \\
& +O\left(h^{1+\delta}\right)
\end{aligned}
$$

for all $h>0$ sufficiently small. Recalling the definition of $E$ this implies

$$
\iint\left(Q^{\prime}\left(L^{-l} f_{0}\right)+E+\gamma L\right) \eta d v d x \geq 0
$$


for all admissible $\eta$, with equality if $\operatorname{supp} \eta \subset \operatorname{supp} f_{0}$ since then also $-\eta$ is admissible. Recalling the definition of $E_{0}$ we obtain

$$
\begin{aligned}
\iint\left(Q^{\prime}\left(L^{-l} f_{0}\right)+E+\gamma L\right)\left(f_{0}+\eta\right) d v d x & \geq \iint\left(Q^{\prime}\left(L^{-l} f_{0}\right)+E+\gamma L\right) f_{0} d v d x \\
& =E_{0} M=E_{0} \iint\left(f_{0}+\eta\right) d v d x
\end{aligned}
$$

or

$$
\iint\left(Q^{\prime}\left(L^{-l} f_{0}\right)+E+\gamma L-E_{0}\right)\left(f_{0}+\eta\right) d v d x \geq 0
$$

again with equality if $\operatorname{supp} \eta \subset \operatorname{supp} f_{0}$. Recalling the class of admissible test functions $\eta$ and the fact that $\epsilon>0$ is arbitrary we conclude that

$$
Q^{\prime}\left(L^{-l} f_{0}\right)+E+\gamma L-E_{0}=0 \text { a. e. on } \operatorname{supp} f_{0},
$$

and

$$
E+\gamma L-E_{0} \geq 0 \text { a. e. on } \mathbb{R}^{6} \backslash \operatorname{supp} f_{0} .
$$

To see the former define $g:=Q^{\prime}\left(L^{-l} f_{0}\right)+E+\gamma L-E_{0}$ and take spherically symmetric, measurable sets $B^{+}, B^{-} \subset \operatorname{supp} f_{0}$ such that $g>0$ on $B^{+}, g<0$ on $B^{-}$, and $g=0$ on $\operatorname{supp} f_{0} \backslash\left(B^{+} \cup B^{-}\right)$. For $\epsilon>0$ define

$$
K_{\epsilon}:=\left\{(x, v) \in \mathbb{R}^{6} \mid \epsilon \leq f_{0}(x, v), L(x, v) \leq 1 / \epsilon\right\} ;
$$

here $g$ and $f_{0}$ are understood as representatives of the corresponding a. e. equivalence classes of measurable functions. Define $B_{\epsilon}^{ \pm}:=B^{ \pm} \cap K_{\epsilon}$, and assume that $\operatorname{vol} B^{+}>0$ and thus also $\operatorname{vol} B_{\epsilon}^{+}>0$ for $\epsilon>0$ sufficiently small. Now define $\eta:=\alpha f_{0}$ on $B_{\epsilon}^{+}, \eta:=-f_{0}$ on $B_{\epsilon}^{-}$and zero elsewhere, where $\alpha \geq 0$ is such that $\int \eta=0$; note that for $\epsilon>0$ sufficiently small, $\int_{B_{\epsilon}^{+}} f_{0}>0$. This $\eta$ is admissable, in particular it has support in the set $K_{\epsilon} \subset \operatorname{supp} f_{0}$. Thus

$$
0=\int g\left(f_{0}+\eta\right)=\int_{B_{\epsilon}^{+}} g(1+\alpha) f_{0}+\int_{\mathbb{R}^{6} \backslash K_{\epsilon}} g f_{0} \geq \int_{B_{\epsilon}^{+}} g f_{0}+\int_{\mathbb{R}^{6} \backslash K_{\epsilon}} g f_{0}
$$

where the first integral is positive and increasing with $\epsilon \rightarrow 0$ and the second converges to 0 . This is a contradiction so that $\operatorname{vol} B^{+}=0$. The same argument works for $B^{-}$so that $g=0$ on $\operatorname{supp} f_{0}$. Thus $0 \leq \int_{\mathbb{R}^{6} \backslash \operatorname{supp} f_{0}} g \eta$ for all admissable $\eta$ which implies that $g \geq 0$ outside $\operatorname{supp} f_{0}$.

This implies that $f_{0}$ is of the form given in the theorem, and by construction

$$
\triangle U_{0}=\frac{1}{r^{2}}\left(r^{2} U_{0}^{\prime}\right)^{\prime}=4 \pi \rho_{0}
$$


so that $\left(f_{0}, \rho_{0}, U_{0}\right)$ is indeed a solution of the Vlasov-Poisson system. Since $f_{0}$ has compact support and $\lim _{r \rightarrow \infty} U_{0}(r)=0$ we conclude that $E_{0}<0$.

\section{Dynamical stability}

We now investigate the dynamical stability of $f_{0}$. First we note that for $f \in \mathcal{F}_{M}$,

$$
\mathcal{D}(f)-\mathcal{D}\left(f_{0}\right)=d\left(f, f_{0}\right)-\frac{1}{8 \pi}\left\|\nabla U_{f}-\nabla U_{0}\right\|_{2}^{2}
$$

where

$$
d\left(f, f_{0}\right)=\iint\left[Q\left(L^{-l} f\right) L^{l}-Q\left(L^{-l} f_{0}\right) L^{l}+\left(E+\gamma L-E_{0}\right)\left(f-f_{0}\right)\right] d v d x .
$$

Theorem 3 Let $Q$ satisfy the assumptions (Q1)-(Q4) and assume that the minimizer $f_{0}$ is unique in $\mathcal{F}_{M}$. Then for all $\epsilon>0$ there is $\delta>0$ such that for any solution $f(t)$ of the Vlasov-Poisson system with $f(0) \in C_{c}^{1}\left(\mathbb{R}^{6}\right) \cap \mathcal{F}_{M}$,

$$
d\left(f(0), f_{0}\right)+\frac{1}{8 \pi}\left\|\nabla U_{f(0)}-\nabla U_{0}\right\|_{2}^{2}<\delta
$$

implies

$$
d\left(f(t), f_{0}\right)+\frac{1}{8 \pi}\left\|\nabla U_{f(t)}-\nabla U_{0}\right\|_{2}^{2}<\epsilon, t \geq 0 .
$$

Proof. We first show that $d\left(f, f_{0}\right) \geq 0, f \in \mathcal{F}_{M}$. For $E+\gamma L-E_{0} \geq 0$ we have $f_{0}=0$, and thus

$$
Q\left(L^{-l} f\right) L^{l}-Q\left(L^{-l} f_{0}\right) L^{l}+\left(E+\gamma L-E_{0}\right)\left(f-f_{0}\right) \geq Q\left(L^{-l} f\right) L^{l} \geq 0 .
$$

For $E+\gamma L-E_{0}<0$

$$
\begin{aligned}
Q\left(L^{-l} f\right) L^{l}-Q\left(L^{-l} f_{0}\right) L^{l}+\left(E+\gamma L-E_{0}\right)\left(f-f_{0}\right) & =\frac{1}{2} Q^{\prime \prime}\left(L^{-l} \tilde{f}\right) L^{-l}\left(f-f_{0}\right)^{2} \\
& \geq 0
\end{aligned}
$$

provided $f>0$; here $\tilde{f}$ is between $f$ and $f_{0}$. If $f=0$, the left hand side is still nonnegative by continuity.

We will use the fact that $\mathcal{D}$ is conserved along any solution $f(t)$ of the Vlasov-Poisson system with $f(0) \in C_{c}^{1}\left(\mathbb{R}^{6}\right) \cap \mathcal{F}_{M}$, i. e., along any classical, 
spherically symmetric solution. This follows from conservation of energy and the fact that both $f(t)$ and $L$ are constant along the measure preserving characteristic flow. Assume the assertion of the theorem were false. Then there exist $\epsilon_{0}>0, t_{n}>0$, and $f_{n}(0) \in C_{c}^{1}\left(\mathbb{R}^{6}\right) \cap \mathcal{F}_{M}$ such that

$$
d\left(f_{n}(0), f_{0}\right)+\frac{1}{8 \pi}\left\|\nabla U_{f_{n}(0)}-\nabla U_{0}\right\|_{2}^{2}=\frac{1}{n}
$$

but

$$
d\left(f_{n}\left(t_{n}\right), f_{0}\right)+\frac{1}{8 \pi}\left\|\nabla U_{f_{n}\left(t_{n}\right)}-\nabla U_{0}\right\|_{2}^{2} \geq \epsilon_{0}>0 .
$$

From (5.1), we have $\lim _{n \rightarrow \infty} \mathcal{D}\left(f_{n}(0)\right)=\mathcal{D}_{M}$. Since $\mathcal{D}(f)$ is invariant under the Vlasov-Poisson flow,

$$
\lim _{n \rightarrow \infty} \mathcal{D}\left(f_{n}\left(t_{n}\right)\right)=\lim _{n \rightarrow \infty} \mathcal{D}\left(f_{n}(0)\right)=\mathcal{D}_{M} .
$$

Thus, $\left(f_{n}\left(t_{n}\right)\right) \subset \mathcal{F}_{M}$ is a minimizing sequence of $\mathcal{D}$, and by Theorem 1 , we deduce that - up to a subsequence- $\left\|\nabla U_{f_{n}\left(t_{n}\right)}-\nabla U_{0}\right\|_{2}^{2} \rightarrow 0$. Again by (5.1), $d\left(f_{n}\left(t_{n}\right), f_{0}\right) \rightarrow 0$, a contradiction.

If $Q^{\prime \prime}$ allows an appropriate bound from below we can obtain an estimate for a weighted $L^{2}$-norm of $f(t)-f_{0}$. If the minimizer $f_{0}$ of $\mathcal{D}$ is not unique in $\mathcal{F}_{M}$ we denote by $\mathcal{M}_{M}$ the set of all minimizers of $\mathcal{D}$ in $\mathcal{F}_{M}$. Then for each $\epsilon>0$ there exists $\delta>0$ such that for any solution $f(t)$ of the Vlasov-Poisson system with $f(0) \in \mathcal{F}_{M} \cap C_{c}^{1}\left(\mathbb{R}^{6}\right)$,

$$
\inf _{f_{0} \in \mathcal{M}_{M}}\left[d\left(f(0), f_{0}\right)+\frac{1}{8 \pi}\left\|\nabla U_{f(0)}-\nabla U_{0}\right\|_{2}^{2}\right]<\delta
$$

implies

$$
\inf _{f_{0} \in \mathcal{M}_{M}}\left[d\left(f(t), f_{0}\right)+\frac{1}{8 \pi}\left\|\nabla U_{f(t)}-\nabla U_{0}\right\|_{2}^{2}\right]<\epsilon, t \geq 0 .
$$

The proof works along the same lines as for Theorem 3 .

\section{Final Remarks:}

(a) The uniqueness of the minimizer $f_{0}$ can be shown in the case of the polytropic ansatz (1.4). In the general case we know of no such result, but we mention that for the argument in Theorem 3 it would suffice if the minimizers of $\mathcal{D}$ in $\mathcal{F}_{M}$ were isolated. 
(b) Obviously we obtain stability only against spherically symmetric perturbations. One reason is that the quantity $L$ is conserved by the characteristic flow only for spherically symmetric solutions. The other is that the splitting estimate (3.4) in Lemma 5 relied on the symmetry which therefore is required even if there is no dependence on $L$.

(c) As was pointed out in the introduction, the polytropic ansatz (1.4) leads to steady states with finite mass and compact support for $k<3 l+7 / 2$. Using a scaling argument as in the proof of Lemma 1 it can be shown that $\mathcal{D}_{M}=-\infty$ for $k>l+3 / 2$, cf. [9], so that our method does not work for this parameter range. The same is true for the methods used in [21] as well as in [18]. Thus $k=l+3 / 2$ seems to be some kind of threshold for the stability properties of steady states. On the other hand it is shown in [1] that the so called Plummer's sphere obtained for $k=3 l+7 / 2$ is the unique minimizer of the total energy of the system under a more restrictive constraint; this model has finite mass but is supported on the whole space $\mathbb{R}^{3}$.

\section{References}

[1] Aly, J. J.: On the lowest energy state of a collisionless selfgravitating system under phase space volume constraints. Monthly Notices Royal Astronomical Soc. 241, 15-27 (1989)

[2] Batt, J., Faltenbacher, W., Horst, E.: Stationary spherically symmetric models in stellar dynamics. Arch. Rational Mech. Anal. 93, 159-183 (1986)

[3] Batt, J., Morrison, P., Rein, G.: Linear stability of stationary solutions of the Vlasov-Poisson system in three dimensions. Arch. Rational Mech. Anal. 130, 163-182 (1995)

[4] Braasch, P., Rein, G., Vukadinović, J.: Nonlinear stability of stationary plasmas - an extension of the energy-Casimir method. SIAM J. Applied Math. 59, 831-844 (1998)

[5] Camm, G. L.: Self-gravitating systems II. Monthly Notices Royal Astronomical Society 112, 155-176 (1952) 
[6] Fridman, A. M., Polyachenko, V. L.: Physics of Gravitating Systems I, Springer-Verlag, New York 1984

[7] Guo, Y.: Stable magnetic equilibria in collisionless plasmas. Comm. Pure and Applied Math. Vol L, 0891-0933 (1997).

[8] Guo, Y.: Stable magnetic equilibria in a symmetric collisionless plasma. Commun. Math. Phys. 200, 211-247 (1999)

[9] Guo, Y.: Variational method in polytropic galaxies. Arch. Rational Mech. Anal., to appear

[10] Guo, Y., Rein, G.: Stable steady states in stellar dynamics. Arch. Rational Mech. Anal., to appear

[11] Guo, Y., Strauss, W.: Nonlinear instability of double-humped equilibria. Ann. Inst. Henri Poincaré 12, 339-352 (1995)

[12] Guo, Y., Strauss, W.: Instability of periodic BGK equilibria. Comm. Pure Appl. Math. 48, 861-894 (1995)

[13] Lions, P.-L., Perthame, B.: Propagation of moments and regularity for the 3-dimensional Vlasov-Poisson system. Invent. math. 105, 415430 (1991)

[14] Pfaffelmoser, K.: Global classical solutions of the Vlasov-Poisson system in three dimensions for general initial data. J. Diff. Eqns. 95, 281-303 (1992)

[15] Rein, G.: Nonlinear stability for the Vlasov-Poisson system-the energy-Casimir method. Math. Meth. in the Appl. Sci. 17, 1129-1140 (1994)

[16] Rein, G.: Nonlinear stability of homogeneous models in Newtonian cosmology. Arch. Rational Mech. Anal. 140, 335-351 (1997)

[17] Rein, G.: Flat steady states in stellar dynamics - existence and stability. Commun. Math. Phys., to appear 
[18] Rein, G., Rendall, A. D.: Compact support of spherically symmetric equilibria in non-relativistic and relativistic galactic dynamics. Math. Proc. Camb. Phil. Soc., to appear

[19] Schaeffer, J.: Global existence of smooth solutions to the VlasovPoisson system in three dimensions. Commun. Part. Diff. Eqns. 16, 1313-1335 (1991)

[20] Wan, Y.-H.: Nonlinear stability of stationary spherically symmetric models in stellar dynamics. Arch. Rational Mech. Anal. 112, 83-95 (1990)

[21] Wolansky, G.: On nonlinear stability of polytropic galaxies. Ann. Inst. Henri Poincaré, 16, 15-48 (1999) 